\title{
"Comunicación política, interpersonal y mediática: aportes para pensar la relación Comunicación, Salud y VIH-sida»
}

\author{
Lic.Milca Cuberli*
}

Recibido: 30 de agosto de 2008

Aprobado: 4 de octubre de 2008

\section{Resumen}

El presente trabajo consiste en una construcción del estado del arte respecto a estudios que involucran la comunicación, la salud y el VIH-sida y que dan un marco posible para un proyecto de investigación cuyo propósito es analizar las características de la dimensión comunicacional implementada en las consultorías de VIH-sida, desde los servicios de la red asistencial pública en la Ciudad de Buenos Aires, Argentine, durante el año 2008. A través de diferentes ejes temáticos se clasifican modalidades diferentes e incluyentes de la comunicación como aporte para el campo de la salud, principalmente desde perspectivas sociológicas y psicológicas. Se indagan aportes de estudios realizados en Argentina y en otros países y se consideran aspectos metodológicos, principales desarrollos conceptuales y resultados en el campo de la comunicación en salud.

\section{Palabras clave}

Comunicación, salud, información, sida

\footnotetext{
* Becaria CONICET (Instituto de Investigaciones Gino Germani-Facultad de Ciencias Sociales /Universidad de Buenos Aires). ccom@mail.fsoc.ubq.ar
} 


\title{
uPolitical, interpersonal, and media communication: Contributions to think about the relationship among communication, health, and HIV-Aids,"
}

\begin{abstract}
This paper presents a construction of the state of the art related to studies which involve communication, health, and HIV-AIDS and provide a possible framework for a research project which purpose is to analyze the characteristics of the implemented communicational dimension in the HIVAIDS consultancies, from the Buenos Aires city (Argentina) health services in year 2008. Different and including communication modalities are classified by topics, as a contribution to the health field, mainly from psychological and sociological perspectives. Contribution of studies conducted in Argentina and other countries are investigated; methodological aspects, especially conceptual developments and results in health communication field are also taken into account.
\end{abstract}

\section{Keys words}

Communication, health, information, AIDS. 


\section{INTRODUCCIÓN}

El presente artículo describe el estado del arte de un proyecto de investigación cuyo objetivo es realizar un análisis comunicacional acerca de prácticas implementadas en las consultorías de información ${ }^{1}$ de VIH/sida (Virus de Inmunodeficiencia Humana/Síndrome de Inmunodeficiencia Adquirida) en la ciudad de Buenos Aires durante el año 2008. Se explorarán las percepciones del equipo de salud respecto a las modalidades comunicacionales y se indagará su relación con las prácticas preventivas y asistenciales implementadas. Las consultorías son postuladas en el marco de una nueva lógica de atención que promueve una relación con el usuario del sistema de salud basada en principios de autonomía, superadora de la histórica y actual inmersa en un modelo denominado médico hegemónico (MMH)².

\section{APORTES DE LOS ANTECEDENTES}

La producción del conocimiento acerca de la relación entre comunicación y salud, llevada a cabo desde diferentes enfoques y perspectivas, muestra la complejidad de su abordaje y lo evidencia como un campo en construcción. Los primeros estudios se remontan a la década del 60, con el interés de conocer qué motiva a las personas para adoptar -0 noconductas saludables (Bandura, 1986, 34). Con una definición polisémica, la comu- nicación en salud evidencia un campo en formación y presenta en la literatura características que la postulan con funciones para la modificación del comportamiento humano y los factores ambientales relacionados con ese comportamiento que, directa o indirectamente, promueven la salud o previenen enfermedades.

Resultados de investigaciones recientes permiten enumerar una serie de factores como:

- Cuestiones derivadas de resultados de experiencias en el campo de la salud que reconocen la comunicación, desde las políticas de salud y desde los servicios, como una estrategia fundamental -junto a otras- para la adquisición de comportamientos saludables y la modificación de prácticas individuales y colectivas;

- Cuestiones propias de las prácticas preventivas implementadas desde los servicios de salud que demuestran la necesidad de trabajar con estrategias como la comunicación en problemáticas y patologías que no presentan procesos curativos, como es el caso del VIH-sida;

- Cuestiones referentes a los equipos de salud que demandan recursos y conocimientos sobre modalidades de comunicación para optimizar los vínculos con las personas que reciben atención y con las comunidades con las cuales se relacionan, en pos de lograr mejores prácticas en salud. Se suman razones que dan cuenta de crisis 
en los modelos de atención y en la organización de los sistemas de salud en cuestiones técnicas, operativas e ideológicas relacionadas con respuestas ineficaces a problemas de salud.

Un primer diagnóstico de situación de este campo puede sintetizarse en los siguientes términos, en un enfoque más tradicional: el objeto casi exclusivo de la investigación ha sido el lugar de la comunicación en las políticas de salud; sus diferentes modalidades y las características de sus componentes, como el diseño y la evaluación; abordaje que vincula la comunicación con la dimensión política. Otras perspectivas han tenido como propósito describir las características de la comunicación, el trabajo con la información y su alcance, la tematización de las problemáticas de salud en diferentes medios de comunicación, su impacto y el estudio de percepciones y conocimientos a través de estudios de opinión pública. En otro plano hay perspectivas que analizan la recepción de mensajes por parte de ciertos grupos etarios y sociales e intentan establecer la relación entre la adquisición de información y los cambios de conductas.

Existen estudios, la mayoría realizados desde la medicina y la psicología, que sitúan a la comunicación en relación con el vínculo entre profesionales del sistema de salud e individuos en calidad de pacientes y/o usuarios de los servicios de salud, donde se analizan dimensiones como la calidad de la atención recibida. Las preocupaciones se sitúan en cuestiones como la información y la comunicación, entre la comprensión de la conducta de los individuos respecto a la enfermedad y las condiciones de la respuesta a la misma que puede visibilizarse en cuestiones como la adherencia al tratamiento. Estos estudios consideran al paciente como sujeto aislado en términos individuales, portador de necesidades que demanda a los servicios las condiciones de su satisfacción. En los últimos años, surge desde las ciencias sociales una forma de pensar los servicios de salud desde una perspectiva de tipo relacional y procesual, que enfatiza la necesidad de sustituir el enfoque médico-paciente por la consideración de una relación más amplia entre población y servicios de salud. Se construye de esta manera una perspectiva que propone incorporar la dimensión de los diferentes grupos sociales y se enfatiza la necesidad de considerar los sesgos producidos por desigualdades de orden cultural, que explicarían cómo se definen desde la perspectiva de los diferentes sujetos, los problemas de salud-enfermedad ${ }^{1}$, a través de lógicas diferenciales entre sí y diferenciales respecto a la lógica de las instituciones del sector salud.

En esta trama de relaciones se inserta un estudio que tiene como objetivo analizar las características que constituyen las prácticas de la dimensión comunicacional presentes en un dispositivo asistencial y preventivo, ofrecido por efectores de salud del primer y segundo nivel de atención y organizaciones (gubernamentales y de la sociedad civil) en el marco de una política de salud implementada por la Coordinación SIDA del Ministerio de Salud de la ciudad de Buenos Aires desde el año 2000. 


\section{CONTEXTUALIZACION DEL OBJETO DE ESTUDIO: VIH-SIDA Y COMUNICACIÓN INTERPERSONAL}

La construcción del objeto de investigación tiene como referente líneas de desarrollo en la investigación sobre la comunicación y sus alcances para la problematización y el cambio de comportamientos desde una modalidad interpersonal. Los estudios sobre este nivel en los servicios de salud vinculan la calidad de la atención médica con aspectos psicoemocionales que se establecen en el vínculo. Problematizan cuestiones en relación con el lenguaje que construye una relación asimétrica en la trasmisión de la información evidenciada en características de poder, de vocabulario técnico o con desconocimiento de los códigos culturales de las personas que concurren a los servicios por parte de los profesionales de salud. Se suman marcos institucionales que que dificultan la producción de relaciones en ambientes íntimos y confidenciales, expresados en factores como la falta de tiempo para desarrollar la consulta y las precariedades del espacio.

El VIH-sida es considerado un caso paradigmático en los problemas de salud por evidenciar y hacer emerger complejidades como el género y las prácticas sexuales, como parte del proceso salud-enfermedadatención que propicia la reestructuración de los servicios de salud y el análisis de prácticas asistenciales y preventivas. Surge la comunicación en sus diferentes modalidades (campañas masivas de difusión, campañas comunitarias, elaboración de materiales de comunicación y/o educación) como un componente sustantivo en las políticas de salud destinadas a atender la problemática.

\section{Tres ejes para reflexionar acerca de la relación entre comunicación y salud}

La clasificación ${ }^{3}$ que se detalla a continuación: comunicación política, comunicación mediática e interpersonal y comunicación interpersonal en el ámbito de la salud responde a criterios clasificatorios operativos, ya que coexisten características compartidas respecto a la comunicación entre los diferentes ejes. Cabe aclarar que en materia del tema de comunicación que ocupa este trabajo, el VIH-sida, la mayoría de los trabajos responde a los dos primeros ejes clasificatorios, por lo tanto, resulta relevante la realización de un trabajo que se propone estudiar la dimensión interpersonal en los servicios de salud en la problemática desde una perspectiva comunicacional. Dimensión que, además, en el campo de los estudios comunicacionales, ha sido la menos estudiada (Guzmán Garza, 2001, p. 33).

\section{a) Eje comunicación y política}

Respecto a la implementación de estrategias comunicacionales y a los escenarios políticos que presentan viabilidad para las mismas, Petracci, Quevedo y Vacchieri (1994) realizan un estudio que evidencia que existe 
una priorización respecto a las problemáticas de salud y su tratamiento mediático en relación con el carácter de su resolución. Analizan comparativamente las estrategias comunicacionales implementadas en relación a las epidemias del sida y del cólera, durante los años 1991-1993 en Argentina. La segunda presenta un escenario político que da lugar a respuestas efectivas en materia comunicacional y de acción política, contrario al tratamiento dado al sida, que postula una comunicación discontinua y confusa, en la que se omiten cuestiones básicas de características preventivas como la utilización del preservativo. Los autores caracterizan esta problemática de salud inmersa en un escenario pre-político, donde el Estado asume un papel pasivo en su tratamiento.

Mónica Petracci y Adriana Viachieri (1994) realizan un estudio de tematización del VIH-sida en la prensa escrita de la Ciudad de Buenos Aires, caracterizándola como una enfermedad mediática. A través del análisis de las campañas en relación con su surgimiento, implementación y contenido, caracterizan los discursos acontecidos por los diferentes actores involucrados: Iglesia, Tercer Sector y Estado y evidencian, de parte de este último, un enunciador que construye discursos «distorsionados» y «contradictorios», basándose en el uso de la metáfora entre otras figuras retóricas, como resultado de la falta de debate acerca de la problemática.

En «Feliz posteridad: cuatro estudios de opinión pública sobre el sida», Mónica Petracci (1994) señala el lugar preponderante de la prevención para controlar la enfermedad. En relación a la misma y a los pro- cesos comunicacionales, propone cuestiones básicas como informar a la población de qué y cómo prevenirse; además de promover la puesta en práctica de los conocimientos informados a partir del análisis de estrategias comunicativas implementadas como política pública. Señala que las mismas se originaron en concepciones erróneas sin poner énfasis en las prácticas sexuales.

Los trabajos de investigación señalan la relación entre las políticas estatales y el tratamiento de los problemas de salud a través del uso de estrategias comunicacionales: describen las falencias que generalmente tienen las mismas y las características de su composición, y analizan a la población como actores destinatarios de los mensajes y el conocimiento sobre cuestiones de salud a través de la indagación de niveles de información que poseen los diferentes colectivos sociales.

\section{b) Eje modalidades de comunicación mediáticas e interpersonales}

Este eje señala investigaciones cuyas ideas centrales son las siguientes: el uso de la comunicación como una dimensión y componente para promover cambios en los comportamientos sociales, la importancia de los medios masivos de comunicación en la difusión de mensajes, y el lugar preponderante de la comunicación interpersonal para trabajar la potencialidad que ofrecen los mismos.

En relación con la interacción que se da entre «pares adolescentes», María Vanda Ianowski (1997) analizó el tipo y nivel de 
información con el que cuentan los jóvenes en colegios secundarios de la Capital Federal y cómo incide en sus comportamientos preventivos. Obtuvo como resultado que la mayoría de los entrevistados conoce los mecanismos de transmisión del sida y las medidas preventivas adecuadas, hecho que coincide con lo que sostienen otras investigaciones que señalan que los jóvenes conocen los mecanismos correctos de transmisión y los medios para la prevención (Nájera, 1990, p. 56). La autora señala también que en relación con las prácticas y el uso del preservativo, no se encuentra correspondencia con el nivel de información poseído, coincidente con investigaciones que señalan que no es causal ni directamente proporcional el nivel de información y las intenciones para llevar a cabo conductas preventivas (Biagini y Sánchez, 1995, 23, 45; Kornblit y Mendez Diz, 1995, p. 67).

Respecto al lugar de los medios de comunicación masivos en «Influencias mediáticas y personales sobre la decisión de protegerse del VIH/ SIDA», Ana Lía Kornblit y Mónica Petracci (2000) analizan las influencias que las personas reconocen en cuanto a su decisión de adoptar o no medidas de protección respecto al VIH. Los datos recogidos muestran que las influencias que las personas reconocen abarcan una doble secuencia, que comprende medios masivos e influencias personales (de profesionales y personas cercanas). En las conclusiones, las autoras señalan que en las políticas preventivas se ha pasado por alto la importancia de los «traductores» de la información. Y que cuando esto se ofrece, se limita a lo transmitido por los medios masivos de comunicación, a excepción de la tarea desarrollada por ONG que trabajan la problemática del sida y ofrecen dispositivos «traductores», olvidando o no reconociedo el papel que como media- dores pueden ofrecer profesionales del equipo de salud, referentes comunitarios u otros significativos con los que los individuos construyen lazos sociales. En una línea similar, a partir de un análisis respecto a las fuentes de información que trabajan la temática del VIH-sida, Muraro y Petracci (2002) hacen, a través de un estudio cuantitativo, una evaluación respecto a los aportes de cada una. Las conclusiones reflejan que las personas acceden a la información mediante procesos de sustitución en la información. En primer lugar, la elección y el acceso a información se produce por fuentes mediáticas masivas y posteriormente por comunicaciones interpersonales caracterizadas por vínculos íntimos o familiares. En una segunda etapa, el acceso de información deviene a través de micromedios o de comunicaciones interpersonales con expertos, distantes de su círculo íntimo.

En «Salud, derecho y opinión pública» Mónica Petracci (2004) trabaja la situación de la opinión pública respecto a la salud sexual y reproductiva en América Latina y el Caribe, los derechos sexuales y reproductivos en Argentina y sus escenarios público-políticos. La autora plantea una perspectiva de la opinión pública integral que contempla el análisis de opiniones, actitudes, valores y representaciones sociales, en oposición a las encuestas como únicas técnicas de relevamiento. En los estudios realizados evidencia la demanda y aceptación de transmisión de información a través de medios de comunicación masivos por parte de diferentes audiencias.

Se demandan investigaciones que trabajen cuestiones referentes a la comunicación interpersonal, como indagar el papel de los «traductores» o «proveedores» de información en marcos intersubjetivos de 
comunicación. En relación con esto, Mónica Gogna (2005) realizó una investigación sobre el estado del arte en materia de sexualidad y derechos reproductivos en Argentina durante los años 1990-2002. En las conclusiones, y como líneas oportunas para investigar, señala en materia de Sexualidad y VIH-sida:

la experiencia de las personas viviendo con VIH; los obstáculos y facilitadores de la comunicación sobre sexualidad entre profesionales de la salud y población usuaria de los servicios públicos de salud (Gogna, 2005, p. 66).

Además, propone como otro tópico a investigar, el uso de los medios de comunicación y la construcción de mensajes en torno a la sexualidad y los derechos sexuales y reproductivos.

\section{c) Eje comunicación interpersonal: equipo de salud y usuarios, relación médico paciente}

A través de un estudio sobre la capacidad por parte del médico de terapia intensiva de comunicar información a la familia del hospitalizado (Rioseco, 2002, p. 23), se problematizan cuestiones como la «comunicabilidad de la información». Bajo una metodología cuantitativa se realizó un estudio descriptivo y prospectivo, donde se tomaron encuestas de opinión aplicadas a familiares de pacientes hospitalizados por un período de 72 horas. Los resultados de este estudio, que toma en sus antecedentes investigaciones respecto a la calidad de la atención, muestran que la familia evalúa la comunicación con el equipo médico, su disponibilidad y cortesía, de mejor forma que lo expresado críticamente en la literatura. Los autores citan que el grupo de familiares con altos grados de escolaridad cuestionan y critican la comunicabilidad señalando aspectos negativos. Este último grupo podría hacer reflexionar acerca de la posición asimétrica que dispensan muchas veces a los usuarios en los servicios de salud, atravesados por un histórico modelo hegemónico donde aquellos sujetos con mayores recursos (simbólicos, educativos, materiales, etc.) pueden posicionarse con autonomía, cuestionar y demandar otras modalidades de atención.

En una línea similar de trabajo, Donabedian (1990), en su problematización del concepto de calidad de la atención médica, trabaja con el componente de la relación interpersonal como aspecto del tratamiento brindado por un profesional. Reconoce como parte de una atención adecuada la accesibilidad, la continuidad y la coordinación, acompañadas del supuesto de que el médico controla la totalidad de los factores que se presentan en una situación de enfermedad. Los supuestos que trabaja el autor en torno al concepto de calidad médica encuentran cuestionamientos en otros autores como Canguilhem (1971) y sus aportes, que mediante elementos críticos cuestiona que el médico pueda controlar los factores presentes en una situación de enfermedad.

Girón (2002) explora la relación entre la calidad de la relación médico paciente y los desenlaces de los encuentros clínicos en servicios de atención primaria en Alicante, España. Se conforman grupos focales integrados por médicos y pacientes, por separado. Desde los primeros, los resultados arrojan una percepción de una relación en- 
tre la pobreza de recursos sanitarios -escaso tiempo de consulta, exceso de carga asistencial, ausencia de recursos específicos, ausencia de formación-, el conflicto con pacientes «difíciles» y la influencia de los factores psicosociales en el encuentro clínico, con el error, el desgaste y la baja calidad de los servicios, relación que es mediada por problemas de comunicación/ exploración en la entrevista clínica y por sentimientos negativos. Los pacientes percibieron una relación entre un funcionamiento caracterizado por consultas masificadas de breve duración, dificultades de comunicación entre médico y paciente, con el error y el conflicto en el encuentro clínico.

Respecto al vínculo profesional de la salud-paciente, Bohórquez y Jaramillo (2004: 8) llevan a cabo un estudio sobre el impacto que tiene en el acto profesional la restricción de los encuentros con el paciente: «encuentros cada vez más técnicos, distantes y fríos que esquivan el diálogo y privilegian la información». Destacan la importancia de la palabra y la necesidad de interpretar en la atención a la corporeidad. Relevan estudios donde la percepción del paciente respecto al médico sugiere más confianza por el conocimiento profesional y científico acerca de su enfermedad y tratamiento, que por la relación de diálogo que pueda establecerse entre los dos.

En «Comunicando malas noticias en Medicina: recomendaciones para hacer de la necesidad virtuds, García Díaz (2006) retoma estudios aleatorizados que han demostrado que el aprendizaje de habilidades de comunicación puede producir un impacto positivo tanto en el dador como en el receptor de malas noticias. Describe un protocolo práctico y dinámico de seis pasos ${ }^{4}$ y propone un mo- delo en el vínculo médico-paciente donde prima la autonomía como una conducta de la relación. Señala la importancia de la entrevista y de tener en cuenta, en la misma, la comunicación no verbal del paciente:

Se entiende por ella: las posturas, expresión facial, tono de voz, aspecto físico, etc. Es necesario identificar contradicciones entre el lenguaje verbal y no verbal, pues ayuda a desvelar dificultades en la comprensión del mensaje (García Díaz, 2006, p. 7).

Propone el uso de lenguaje sencillo para comunicar información al paciente y el uso de material gráfico como apoyatura en la entrevista. La conclusión que transmite el autor se resume en considerar la evidencia de otras investigaciones que señalan también, que la implementación de un enfoque humanístico y protocolizado y el aprendizaje de ciertas habilidades de comunicación pueden mejorar la relación médico-paciente.

Estudios respecto a la relación interpersonal entre médicos y pacientes y su influencia en la adherencia al tratamiento (Serra Rexach, 2003, p. 12; Rost y Roter, 2006, p. 35) concluyen en sus resultados que, para mejorar la relación y conseguir una mejor evolución de las enfermedades, se debe considerar: el punto de vista del paciente, sus aspectos psicosociales, valorar las limitaciones sensoriales y funcionales e implicar a los cuidadores en el diagnóstico y en el plan de cuidados. Reconocen la entrevista como un espacio donde privilegiar el discurso del paciente. Existe un consenso en la literatura que en la relación médico-paciente existe de parte del primer actor una mirada profesio- 
nal que se distancia de la realidad concreta del individuo-paciente, que por su posición muchas veces se encuentra en un estado de vulnerabilidad. Esta concepción negaría el componente subjetivo de la enfermedad, estableciendo una relación entre el médico y la enfermedad del sujeto, que no tiene en cuenta al sujeto que padece la misma (Canguilhem, 1971, p. 12, p .67). De la relación entre ambos actores surge información valorizada y transformada en diagnóstico, que se define también en el universo de las representaciones del paciente.

La mayoría de estos estudios utilizan metodologías cuali-cuantitativas mediante la realización de cuestionarios. Reconocen dificultades en la relación profesional de la salud-usuarios y su impacto en los tratamientos y desarrollos de las patologías de salud. Privilegian la comunicación verbal y su reconocimiento en el encuadre de atención por parte del profesional.

\section{REFLEXIONES}

En relación con el objeto que se propone para esta investigación y priorizando la relación interpersonal profesionales de la salud-usuarios, no se han encontrado trabajos de investigación que aborden el vínculo desde una perspectiva comunicacional, específicamente en relación con la problemática del VIH-sida. Se propone un análisis que contemple una noción de comunicación que aborde en un sentido procesual, interaccional y de mediaciones la relación establecida desde los servicios de salud, entre personas integrantes de los equipos de salud y usuarios del sistema. Compren- der la comunicación, más allá de los medios o instrumentos de transmisión, es afrontarla como «un elemento estructural de la sociedad» (Torrico Villanueva, 2004, 18) en el que se interrelacionan los individuos, las culturas y sus modalidades de intercambio. Pensar la comunicación de esta manera implica afirmar que existen múltiples formas en las que el hombre se comunica, ya que todo comunica, pero que también existen reglas que regirán la interacción. Se propone una perspectiva de la comunicación como constitutiva de un proceso de producción de sentidos de carácter social. Surge el reconocimiento de los diferentes individuos que interactúan con sus representaciones, opiniones y conocimientos, en y desde las prácticas y la información en salud. Los interrogantes que guían el presente estudio problematizan cuestiones que competen a los procesos de información y comunicación en el marco de ámbitos de la salud por parte de los miembros del equipo de salud, y que se relacionan con sus concepciones acerca de los modelos de atención.

Se hace una primera referencia en este espacio de asesoramiento a interacciones efectivas entre miembros del equipo de salud y usuarios. El desarrollo de relaciones caracterizadas por supremacía de la información por sobre la comunicación, puede acentuar relaciones asimétricas entre miembros del equipo de salud y usuarios, lo que impactaría de modo negativo en los aportes que podría hacer la comunicación interpersonal en relación con la promoción para el uso del preservativo, la adherencia al tratamiento en caso de infecciones por $\mathrm{VIH}$-sida o el testeo a la pareja sexual del consultante, entre otras. 


\section{NOTAS}

1 Las consultorías denominadas CePAD -Centros de Prevención, Asesoramiento y Diagnósticopermiten un trabajo local de características preventivas y asistenciales que promueven asesoramiento en el marco de una comunicación interpersonal. Son una estrategia implementada junto a otras, para disminuir la transmisión del VIH en la ciudad de Buenos Aires e intentan presentar un modelo alternativo para la realización del test de VIH, único modo de conocer hasta el momento la presencia del virus en la sangre.

2 Eduardo Menéndez (1988) caracteriza el Modelo Médico Hegemónico (MMH) como: «un conjunto de prácticas, saberes y teorías generados por el desarrollo de lo que se conoce como medicina científica, el cual desde fines del siglo XVIII ha ido logrando establecer como subalternas al conjunto de prácticas, saberes e ideologías teóricas hasta entonces dominantes en los conjuntos sociales, hasta lograr identificarse como la única forma de atender la enfermedad legitimada tanto por criterios científicos, como por el Estados. El modelo $\mathrm{MMH}$ asume la enfermedad, como un hecho natural y biológico, no como un hecho social e histórico. Si lo incluye, sólo lo hace en la explicación de los problemas, no en su abordaje.

3 La producción académica en Argentina sobre el campo de la comunicación y la salud en relación al VIH-sida refiere en su mayoría a trabajos de investigación de opinión pública, comunicación política, tematización de la problemática en medios gráficos y análisis de campañas. Respecto a la comunicación interpersonal, la literatura encontrada versa sobre trabajos nacionales e internacionales respecto a diferentes patologías, incluido el VIH-sida, en el campo de la salud pública.

4 Este protocolo, ideado por Amy Bruckman ha sido validado en investigaciones respecto a la comunicabilidad de resultados médicos, diagnósticos y tratamientos en patología de cáncer genitomamario.

\section{REFERENCIAS BIBLIOGRÁFICAS}

Bandura, Albert. 1971. Social Learning Theory. Nueva Jersey: Prentice Hall Englewood Cliffs, 34.

Biagini, Graciela, Sánchez, María. 1995. Actores sociales y SIDA. Las organizacones no gubernamentales en Argentina y el complejo VIH-sida. Buenos Aires: Espacio Editorial, 23, 45.

Bohórquez, Francisco; Jaramillo Luís. 2005. El diálogo como encuentro: aproximaciones a la relación profesional de la salud-paciente. En: Revista INDEX de Enfermería Digital No. XIV; Otoño Vol 50. Madrid, 8. Disponible en http://www.index-f.com/index-enfermeria/50revista/50_sumario.php. Fecha última consulta en Internet 15-01-08.

Canguilhem, G. 1971. Lo normal y lo patológico. Buenos Aires: Editorial Siglo XXI, 12, 67.

Anagramas, Volumen 7 No. 13, pp. 77-89 • ISSN 1692-2522 • Junio/diciembre de 2008/232 p. Medellín, Colombia 
Donabedian, Avedis. 1984. La calidad de la atención médica. Definición y métodos de evaluación. México: La Prensa Médica Mexicana.

García Díaz, Francisco. 2006. Comunicando malas noticias en Medicina: recomendaciones para hacer de la necesidad virtud. En: Medicina Intensiva Vl $30 \mathrm{~N}^{\circ}$ 9. Madrid 7. Disponible en http://cat.inist.fr/ ?aModele $=$ afficheNEcpsidt $=18387709$. Última consulta 18-01 08.

Giron, M; Begoña, B; Medina, E. y Taler, MS. 2002. Calidad de la relación médico paciente y resultados de los encuentros clínicos en atención primaria de Alicante: un estudio con grupos focales. En: Revista Española de Salud Pública Vol.76 N.5 Oct Madrid. Disponible en http:// www.scielosp.org/scielo.php?script=sci_arttextEpid=S1 135-57272002000500016. Última con sulta en Internet 18-01-08.

Guzmán Garza, María. 2001. Comunicación Interpersonal, primer reto del profesional de la comunicación. Ponencia Congreso de Comunicación México DF, UNAM, 33.

Ianowski, María Vanda .1997. La escuela secundaria como espacio privilegiado en la articulación de campañas preventivas. En: SIDA y Sociedad. Buenos Aires: Espacio Editorial.

Nájera, Paul .1990. Educación para la salud en el caso del SIDA. En Rop Nájera (ed.) SIDA: de la Biomedicina a la Sociedad. Madrid: Editorial Eudema, 56.

Kornblit, Ana Lía; Petracci, Mónica. 2000. Influencias mediáticas y personales sobre la decisión de la gente de protegerse del VIH/SIDA. En: Kornblit, Ana Lía; SIDA: entre el cuidado y el riesgo. Estudios en población general $y$ en personas afectadas: Buenos Aires: Alianza Editorial.

Kornblit, Ana Lía; Mendez Diz, Ana María .1995. Los trabajadores de la salud en los tiempos del SIDA. Centros de Estudios Avanzados. Oficina de Publicaciones del Ciclo Básico Común. Buenos Aires: Universidad de Buenos Aires, 67.

Gogna, Mónica. 2002. Estado del Arte. Investigación sobre sexualidad y derechos en la Argentina (19902002). Buenos Aires; CEDES-CLAM, 66.

Menéndez, Eduardo .1988. Morir de alcohol, saber y hegemonía médica. México D. F.: Ed. Alianza Mexicana.

Méndez, Eduardo 2003. Modelos de atención de los padecimientos: de exclusiones teóricas y articulaciones prácticas. En Spinelli H (compilador) Salud Colectiva. Buenos Aires: Lugar Editorial.

Petracci, Mónica; Quevedo, Luis; Vacchieri, Adriana. 1994. Los modelos políticos-comunicativos del SIDA y el Cólera en la Argentina. 1991-1993. Buenos Aires: Artículo correspondiente a una investigación perteneciente al Programa OPS-CLACSO de Promoción de la Investigación Social: Estado y Salud en América Latina y el Caribe.

Petracci, Mónica. 1994. Feliz posteridad. Cuatro estudios de opinión pública sobre el SIDA. Buenos Aires: Ediciones Letra Buena. 
Petracci, Mónica .1994. «SIDA, medios de comunicación y campañas» En Feliz posteridad. Cuatro estudios de opinión pública sobre el SIDA. Buenos Aires: Ediciones Letra Buena.

Petracci, Mónica; MURARO Heriberto.1998. Circuitos comunicacionales de información sobre VIH/SIDA». Cuaderno de la Cátedra Técnicas de Investigación en Opinión Pública y Mercado. Carrera de Ciencias de la Comunicación. Facultad de Ciencias Sociales, Universidad de Buenos Aires.

Petracci, Mónica. 2004. Salud, derecho y opinión pública. Buenos Aires: Grupo Editorial Norma.

Rioseco, F; Jarpa, Ma. E; Valenzuela, C. 2003. Evaluación de la comunicación entre el equipo médico y los familiares de los pacientes hospitalizados en UCI. En Revista Chilena de Medicina. $\mathrm{N}^{\circ} 6$.

Disponible en http://bases.bireme.br/cgibin/wxislind.exe/iah/LILACS/lang. Última Consulta en Internet 25-02-08.

Serra Rexach, JA .2003. Comunicación entre el paciente anciano y el médico. Ponencia en Anales de Medicina Interna, Madrid, 12.

Rost, K; Roter, D., 2006. Predictors of recall of medications regimens and recommendatios for lifestyle change in elderly patients. Baltimore: Gerontologist Group, 35.

Torrico Villanueva, Erick. 2004. Abordajes y períodos de la teoría de la comunicación. Buenos Aires: Grupo Editorial Norma, 18. 